\title{
Urgences
}

\section{Parler maître : Lacan, le signifiant nomade et le diable calembourgeois au corps des Sciences humaines avec un petit aperçu joycien}

\section{Jean-Pierre Vidal}

Numéro 30, décembre 1990

L'autre du texte

URI : https://id.erudit.org/iderudit/025622ar

DOI : https://doi.org/10.7202/025622ar

Aller au sommaire du numéro

Éditeur(s)

Urgences

ISSN

0226-9554 (imprimé)

1927-3924 (numérique)

Découvrir la revue

Citer cet article

Vidal, J.-P. (1990). Parler maître : Lacan, le signifiant nomade et le diable calembourgeois au corps des Sciences humaines avec un petit aperçu joycien. Urgences, (30), 18-30. https://doi.org/10.7202/025622ar d'utilisation que vous pouvez consulter en ligne. 


\section{Parler maître: Lacan, le signifiant nomade et le diable calembourgeois au corps des Sciences Humaines avec un petit aperçu joycien Jean-Pierre Vidal}

Quiconque s'avance vers les mots s'offre à devenir un mot qui s'ajoutera aux autres offre démente mais qui, si elle s'énonce avec assez d'intensité, peut produire ce résultat que, au coeur de la démence, la perdition', nécessaire perdition, et le salut, impossible salut, l'un à l'autre se soudent.

Giorgio Manganelli, Discours de l'ombre et du blason, coll. * Fiction \& Cie», Paris, Seuil, 1987, p. 152.

Les temps intellectuels sont à la palinodie oublieuse; l'inconséquence et l'ignorance, plus encore qu'un cynisme à l'ironie et au courage heureusement hautains, fondent, en l'ère postmoderne où nous voici tapis, la pleutre convivialité d'un consensus mollasson. Systémique, holistique, droits tous azimuts, une gigantesque implosion d'obscénité théorique est venue effondrer la galerie. Dans la médiatisation gloutonne qui s'est définitivement refermée sur tout ce qui d'art, de culture, de littérature, bougeait encore hors de l'engeance accommodante, pandigéreuse et surexcréteuse de la communication, le babil flotte, imbécile et satisfait, sur les eaux, sur la soupe communes.

Parfois quelqu'un vient y cracher, vitupérateur magnifique, feu Thomas Bernhard, entre si peu d'autres, de moins en moins, de loin en loin.

Tel jadis le grand Pan, il semble bien crevé lui aussi le persévère, l'imprécateur du « je m'ovalise», le tribun de la fondatrice dérive des signifiants.

Puisqu'ainsi donc au désert l'ouvre-verbes, le colle-noms qu'il fut est maintenant passé, parlons encore un peu de lui au présent de la résistance ringarde. 
En Lacan, en effet, en la pratique de Lacan - car il n'a jamais cessé de souligner que son exercice et de la psychanalyse et de la parole «magistrale * étaient sinon la même chose du moins deux modes inséparables de son rapport au savoir et au langage - se vivent et tôt s'exemplarisent les diverses problématiques qui sous-tendent les Sciences dites Humaines qu'il proposait, on s'en souviendra, de nommer plutôt * conjecturales $* 1$. Conjecturales parce que leur existence même tient à une contrainte majeure, leur fondation du même coup, que l'on pourrait formuler ainsi: comment penser de l'intérieur du langage et par lui ce que l'on doit poser comme radicalement étranger à lui. En une formule encore plus lapidaire: comment penser son propre dehors et le corollaire: comment poser son improbable dedans.

On le voit, prises sous cet angle, dès le départ les Sciences Humaines sont aux prises avec le problème de l'Autre et l'Autre n'a d'autre peau, d'autre seuil, d'autre marge que le langage. Le fantasme des Sciences Humaines c'est l'atteinte de l'Autre hors du langage, pourtant son seul lieu d'émergence, et c'est un paradoxe, car le trouver enfin ce serait peut-être devenir science mais certes plus humaine. Ce serait surtout y perdre la voix.

Trouver l'Autre pour s'y inféoder et en tirer une légitimité rassurante, par l'épiphanie définitive d'un * réel» où se fonderait le symbolique, enfin loi absolue, c'est-à-dire liquidation de l'imaginaire, trouver l'Autre par un tel sacrifice de la parole au métalangage des gogos ou des pédagogues pressés ce serait trouver la mort, son silence vide, son silence comme surface sans aspérité d'une contingence définitive, "puisque une vérité qui parle a peu de chose en commun avec un noumène qui, de mémoire de raison pure, la ferme ${ }^{2}$. Il faut entendre ici que toute vérité, qui n'est toujours que de parole, qui toujours «se fonde de ce qu'elle parle et [...) n'a pas d'autre moyen pour ce faire ${ }^{3}$, que toute vérité est bornée par le silence fondamental de la "chose en soi * kantienne qui, argotiquement, au plus loin que la raison la peut aller débusquer, se tait ( «la ferme ") et clôt, délimite, enchâsse même la vérité ( la ferme» encore mais dans un niveau de langage plus

\footnotetext{
1 Cf. * La science et la vérité" in Ecrits II, coll. « Points", Paris, Seuil, 1971, $244 \mathrm{p}$.

2 Ibid., p. 234.

3 ibid., p. 233.
} 
relevé). D'où il s'ensuit que la science, occupée, dirait-on, à décrire (c'est-à-dire changer) le noumène, n'atteint jamais la vérité mais s'articule à elle par le * sujet * qu'elle postule, sujet qui fonde la psychanalyse puisqu'en lui, et en lui seul, se joue "la vérité comme cause». Car c'est bien parce que le symbolique n'est pas plein, total, refermé, qu'il y a de l'imaginaire, du langage et de l'homme. Nous naissons, jour après jour, d'une faille. L'homme est la mesure, sinon de toutes choses, au moins du symbolique sans quoi il ne saurait être. Car son imaginaire scande le rythme du monde où il se débat en tous sens, et notamment du terme. En fait, l'homme est le rythme du monde avant même d'en être le sens.

Ce fantasme donc d'un être hors langage qui fonde les Sciences Humaines ne serait-ce pas la postulation que savoir et discours ont des "topoï " distincts ou du moins distinguables? Or, loin que cette distinction qui est en elle-même une fiction sans doute nécessaire puisse prendre la forme musclée d'une séparation absolue telle que la rêvent certaines Sciences Humaines (toutes celles, en particulier, qui grenouillent aux abords de la littérature), dans leur rêve archaïsant d'un modèle tiré des vieilles Sciences de la Nature, les sciences actuelles la postulent plutôt comme un "pli «, au sens que ce terme revêt dans la théorie des catastrophes de René Thom où, rappelonsle, il désigne ce lieu (et ce processus) dont chaque élément qui le forme appartient à au moins deux surfaces ou deux structures "distinctes » mais solidaires, au sens étymologique. L'exemple le plus souvent cité de ce type de «pli n c'est, ô Saussure, celui des deux côtés d'une feuille, de papier par exemple.

Ainsi la réflexion que mène Lacan, à partir de Freud, sur les déterminations réciproques, quant au sujet, du dehors et du dedans, sous les espèces du « pli » où se départagent en même temps mais selon des modalités diversifiées discours et savoir (ou, si l'on préfère, vérité et science), somatique et sémiotique, inconscient et langage, permet de penser comme passage d'une matière jamais purement matérielle à une idéalité jamais tout à fait abstraite, d'une part la naissance du sujet mais aussi les phénomenes culturels qui sont du réel - et le réel est pour Lacan, rappelons-le, non pas même un impossible mais l'impossible, "tout simplement ${ }^{4}-t e l$ 
qu'un imaginaire, individuel socialisé ou socialement individualisé, on ne sait, s'y projetant et s'en distanciant, les organise en symbolique. A ce niveau, la mimesis est donc à penser en rapport avec le stade du miroir. Et le geste qui la fonde est la deixis d'un regard renvoyé à lui-même.

Renvoyé à peine posé car c'est le lieu qui l'ordonne. Tel le miroir commandant au sujet d'être enfin où naguère, juste avant ce regard risqué en coin, s'agitaient vagissant les grouillements vains d'inopinés clivages: *Wo es war, soll Ich verden *, * où c'était il me faut advenir . Ou, puisque le miroir est un labyrinthe, mon lieu d'émergence est le futur que le passé de l'Autre ( le «Es», le * ça * innommable) préparait. Car, de même que c'était, dans le commentaire qu'en donne Lacan, l'» ergo * de la loi qui fait surgir l'« ego sum * du * cogito * cartésien, en une suite que ne trouvera étonnamment paragrammatique que qui ne sait ce que parler veut dire en fait de vérité, de même est-ce le ulégisigne " (pour parler peircien) de ce * soll... werden » qui fait affleurer le «Ich». Le «je» est ainsi toujours l'effet irrémédiable d'une syntaxe imparable. La syntaxe du monde, mais seulement telle du moins qu'en le miroir je la puis me voir faire voir me faire. Nulle faute de frappe, il faut ici en prévenir, dans cette dernière formulation: le redoublement y traduit la *double contrainte " où se trouve fait et refait (ou * faitt * en québécois) le sujet. Il n'est que de se voir être et il ne voit que d'être. Même, il ne voit que de l'être, comme on ne voit que du feu: une illusion ici le consume.

Toute pensée de la mimesis qui, comme on le voit le plus souvent, se fonde sur la coupure plus que sur le pli, méconnaît la solution de continuité qui fait tenir ensemble comme instances séparées, mais l'une par l'autre, le regard, le miroir et tout ce qui s'ensuit. C'est-à-dire l'énumération de ce qui s'y voit comme par l'effet d'un * on ", la signature apposée en retour sur qui s'y voit « je * irréductible enfin, la détermination du lieu « elle * rétrospectif (la mère, la mémoire, l'Histoire, c'est tout un: c'est ce qui vient après et précisément parce que c'est ce d'où toujours on vient, d'où toujours on n'en finit tellement pas de venir que ce lieu improbable d'une origine insensée devient un après-coup lui-même) d'où ce *je» se voit dès lors parti tout autant qu'il s'y voit devenu l'autre du

en tant que le réel c'est l'impossible, tout simplement." «C'est à la lecture do Freud... ", texte inédit de Lacan, in Robert Georgin, Lacan, coll. " Cistre", Lausanne, L'Ảge d'homme, 1977, p. 14-15. 
"quelqu'un* qui l'occupe encore. C'est dans une telle spatialisation du sujet que, par exemple, la *chora* sémiotique de Kristeva ${ }^{5}$ s'oppose ainsi, «maternelle ", au «topos « cette autre façon grecque de dire le lieu (mais le lieu, dans ce cas, de la loi) parce que notamment la langue grecque y dit, selon Bailly, à la fois l'* espace de terre limité et occupé par quelqu'un ou par quelque chose * et l'* espace de terre situé entre deux objets*. La * chora* ainsi entendue, on le voit, mine tout * topos* pris comme coïncidence parfaite, tout propre donc, que ce soit de sens ou d'homme. Elle est le lieu du " au lieu de * sans cesse, de la substitution permanente, de l'alternative, de l'altérité en un mot, mais une altérité en mouvement toujours, une altérité qui serait donc le mode propre d'émergence de la conscience ou, comme dirait Lacan qui y tenait sans doute un peu par bravade, du * cogito* en tant qu'un * ergo* le fait effet de lui-même (tel ce regard en le miroir) et non cette altérité de foire, ce dérisoire hochet où nos contemporains trop souvent adorent à peu de frais leur bonne conscience si vite quiète. La * chora", comme l'inconscient, n'a ainsi d'autre lieu que la constellation toujours déportée de la différance. Elle se devrait donc sans doute désigner du titre que Michel Butor, dans un geste fort lacanien, barre en couverture d'une de ses oeuvres majeures: $O \mathbb{A}^{6}$.

Il est en effet dès lors question de quelque * esprit du lieu ».

Ou du virus que Jacques Lacan, structuraliste certes mais structuraliste dynamique, riva au mitan du corpus des sciences conjecturales, comme l' éternelle ironie" du vieil hegelien qu'il ne cessa jamais d'être.

Ironie, effet maître (et non, bien sûr, effet du maître, ce qui serait, à proprement parler, le contraire), c'est le jeu du sujet en son aire, du sujet qui se saisit et du même coup se déprend, se distingue, tout uniment de lui-même et du monde. C'est la leçon du lieu, celle du miroir, celle du signifiant, y compris linguistique, du moins tel qu'un Lacan, par exemple («... l'écrivain qui de son style marque la langue, je sais à qui je pense. ${ }^{7}$ Parbleu! Il n'y a, en l'occurrence, pas que Joyce ou Duras), incessamment la délivre.

5 La révolution du langage poétique, coll. « Tel Quel», Paris, Seuil, 1974, $645 \mathrm{p}$.

6 Paris, Gallimard, 1971, $395 \mathrm{p}$.

7 Ibid., p. 223. 
C'est la leçon de cette faille qu'ouvre entre métonymie et métaphore la tmèse ${ }^{8}$ d'où surgit la parole oraculaire comme la voix même de la vérité, nue d'être inouie, fracas obscène de la force des choses, * stratégie fatale * au sens que Baudrillard voue à un tel oxymoron.

La tmèse, parler maître parce que projection née du suspens, "supplément de copule " dirait Derrida. La tmèse produit de la mise en scène du monde lorsque le rideau s'ouvre sur l'humain qu'elle force à sortir enfin des coulisses. La tmèse, notre mère.

Ainsi que la métaphore, saut éperdu dans le symbolique enfin, naît du tissage métonymique d'absence qu'ourdit incessamment l'imaginaire, la parole de l'oracle jaillit enfin, tmèse, des lignes de fuite du lieu, comme un ordre insensé, une convocation folle, un impossible réel, qu'une manière de thérapeute, ou plus nettement encore d'analyste, infléchit, civilise, transcrit et fait accéder au sens.

Il n'y a donc de sens que critique, de signification que. dans l'après-coup, dans le social de la parole détournée, apprivoisée. Et de même que l'oracle commande son interprète, comme en une deixis inverse où ce serait le monde qui désignerait l'homme, de même le discours en tant que vérité du sujet commande-t-il au sens que la communication en tire.

Or tout artiste - et Lacan en fut un, sans conteste - se fait être a la fois le lieu de l'oracle et de son interprète, du discours et de son sens, et la tmèse de ce *à la fois", c'est l'oeuvre, non-lieu que l'homme (dire "ou la femme* ici n'aurait pas grand sens. Aussi faut-il l'entendre elle-aussi, la biographique du moins, dans cet *un ") tend au monde pour. qu'il s'y prenne et y fasse naître le sujet. Tout art est ainsi une rhétorique du non-lieu.

L'artiste, comme le shaman, comme le psychanalyste qui professe ou comme le professeur tout court, espèce rare en nos temps de syllabus obligatoire, accepte d'être le jouet $e t$ l'enjeu d'une parole qui le traverse et le rive au monde. Mais il lui faut mobiliser tout son art, rester tendu à l'extrême

8 Cf. encore Giorgio Manganelli, Discours de l'ombre et du blason, p. 210: "Sois-en sûr: je n'ai pas d'idées. Mais je suis expert en nonsense, limericks. lipogrammes et chants alphabétiques. Les mots sont des amis qui ne me déçoivent jamais. Et j'ai une tendresse toute particulière pour la tmèse. Elle est la figure de la lacération, l'hiéroglyphe du "dividit"." 
24

pointe de lui-même, de son «métier ", de sa ruse, de sa sincérité aussi et de la modestie qu'elle lui commande, pour parvenir à faire parler par lui quelque chose dont la seule chose qu'il puisse en dire c'est qu'il ne sait pas ce qu'il dit quand elle parle.

Ainsi de Jacques-Marie Lacan, maître-rhéteur, orateur oraculaire, parleur de vérité qui joua gros le théorique comme s'il n'était que de la lettre, ce qu'en fait on finit par le savoir être, si l'on suit sa leçon.

Mais suivre la leçon de Jacques-Marie Lacan, passer par son séminaire, qu'est-ce que, dès lors, cela peut bien vouloir dire, et pour qui? Autrement dit, à qui parle le shaman? Et que se dépose-t-il ailleurs de sa parole, après l'effervescence?

Poser ainsi la question c'est égrener, chapelet identitaire, indistincts grains d'être, «les non-dupes errent», où quelque Crétois encore s'agite en son paradoxe. Car de même que le fameux "je dis que je mens" produit une double contrainte (sauf pour les petits malins de la philosophie analytique) le non moins fameux "les non-dupes errent » joue de la différence qu'un contradictoire de cet ordre et de cette magnitude inévitablement installe. En effet, s'ils errent précisément de ne pas être dupes, ou s'ils ne sont pas dupes précisément parce qu'ils errent, comme, dans un autre discours, sont aveugles ceux qui voient, c'est que la vérité, comme le royaume des cieux aux faibles en esprit, n'est accessible qu'aux dupes. Précisément parce qu'elle est la vérité, précisément parce qu'ils sont les dupes. Mais dès lors dupes de quoi? Du père, pardi ! Pas du petit papa, parbleu! De la configuration père, fin mot de la farce, nom majuscule et déchu. En ce jeu de * qui perd gagne * de *l'entreprêt» qui va " de ce qui perdure de perte pure à ce qui ne parie que du père au pire ${ }^{9}$.

Ce n'est qu'en autant que la «perte pure», telle que le stade du miroir la révèle comme le paradoxe qu'elle est (car, en toute logique, une perte ne saurait se dire "pure" dans la mesure où elle doit bien être perte de quelque chose, tirer donc part - et parti - de ses qualités de ce dont elle est la perte), n'en finit pas d'être, *éternel présent \# de l'anamnèse, répercutée à travers le sujet au point qu'il n'est en lui écho que d'elle, que cet écho, même, est l'"ergo " de son " ego sum ",

Télévision, Paris, Seuil, 1973, p. 72. 
ce n'est qu'en autant que la perte tient à jamais le sujet qu'il peut, qu'il doit se parier un père ou pire, c'est-à-dire "risquer tout ", risquer de remonter jusqu'à ce qui, de toute éternité, le comprend et l'efface. La perte lui a donné lieu, un temps de mort risque de lui donner fin, pire même que la fin, un jamais lieu. Et pourtant c'est cela qu'il doit sans cesse affronter pour assurer son ordinaire: le risque de la structure, d'une structure qui est l'ossification, la stase monumentale de l'Autre, autant dire «l'uom di sasso, l'uomo bianco ${ }^{10}$, pétrification d'os qui vient saisir le trop vif Giovanni.

Pour l'artiste, l'oeuvre à risquer est comme l'ombre portée de la statue du commandeur. C'est en ce sens que l'on a pu dire qu'il est toujours le fils de ses oeuvres, en ce sens aussi que Joyce a pu développer, dans Ulysses, le célèbre paradoxe qui fait de Shakespeare, pris comme un oracle aux rets d'un espace ici scénique, à la fois le fils d'Hamlet et le père de son père et donc à lui-même son propre père. Ainsi parier le père ici devient naturellement jouer le fantôme pour faire venir «du» fils. Qu'on en juge plutôt par cet extrait exemplaire dans l'ironie qui le sous-tend:

The play begins. A player comes on under the shadow, made up in the castoff mail of a court buck, a wellset man with a bass voice. It is the ghost, the king, a king and no king, and the player is Shakespeare who has studied Hamlet all the years of his life which were not vanity in order to play the part of the spectre. He speaks the words to Burbage, the young player who stands before him beyond the rack of cerecloth, calling him by a name: " Hamlet, I am thy father's spirit ", bidding him list. To a son he speaks, the son of his soul, the prince, young Hamlet and to the son of his body. Hamnet Shakespeare, who has died in Stratford that his namesake may live for ever. ${ }^{11}$

Venu de l'ombre, accoutré du rebut guerrier de quelque bellâtre courtisan (littéralement " de la cotte de mailles abandonnée par un mâle de la court"), comme s'il devait ainsi se représenter lui-même en rejeton déjeté de quelque figure de fils, mais bien découplé ("a wellset man"), très présent de toute sa voix de basse, voici donc le fantôme, le roi, un roi qui n'est nullement roi (on pourrait aussi traduire: «un roi et en 
même temps pas roi $\rightsquigarrow)$, autant dire une vérité, quelque chose qui ne se soutient que de parole, voici le fantôme et l'acteur, mieux, celui qui joue est Shakespeare * qui a étudié Hamlet pendant toutes les années de sa vie qui ne furent pas vanité dans le but de jouer le rôle du spectre * 12 . Le père ici, on le voit de reste, n'est qu'un parcours et une ascèse. Le père, le fût-on soi-même, on ne peut que le jouer, l'incarner mais toujours $a$ côté de son lieu, de la plaque cénotaphe qui toujours le commémore comme une absence nécessaire (il n'est de père que manqué, n'en déplaise à certain psychologue à la mode médiatique), le jouer à côté de son lieu et à l'écoute du fils, l'écoute du désir (et il n'est même pas nécessaire d'ajouter « du fils * tant il n'est de désir que du fils). Tout ce qui ne fut pas vanité dans la vie du grand Will se résume à cette écoute du désir de l'oeuvre, afin de pouvoir, modestement, y tenir sa partie. Ainsi l'artiste se fait-il à la fois lieu de l'oracle et de son interprète, comme nous le disions plus haut.

Le père, donc, n'existe que comme absence (c'est la condition même de la vie) mais il faut savoir rester dupe de l'imposture de cette existence pour ne pas errer, pour marcher droit. Le père comme leurre est le nord qu'indique, si quelque fausse science, quelque * pire * ne vient pas la dérouter, la boussole que devient, tout entier, le sujet, d'être, né de la perte pure, un pur désir, une tension, un mouvement, autrement dit, d'une formule que l'on voudrait furieusement polysémique: une raison d'être. Le sujet n'a pas de raison d'être, il est raison d'être. En autant qu'il parie « du» père, comme sa propre signature de la perte qui l'a lancé au monde. Ou, en termes joyciens, dans la suite du discours de Dedalus sur Shakespeare: "... through the ghost of the unquiet father the image of the unliving son looks forth ${ }^{13}$. Que l'on pourrait traduire: «à travers le fantôme du père sans repos c'est l'image du fils sans vie qui regarde devant, qui regarde lavenir * " to look forth " contient cette nuance prospective qui l'apparente à « to look forward $»$ : attendre avec impatience, anticiper avec plaisir). C'est le désir du fils qui ici voit et va devant, se projette, à travers un insaisissable fantôme de père nomade.

Car le sujet n'est plein que de la relance de la perte initiale qui au miroir le constituait, relance effectuée par ce pari

12 Traduction d'Auguste Morel, revue par Valéry Larbaud, Stuart Gilbert et Joyce, coll. * Folio", Paris, Gallimard, tome I, p. 275.

13 Ulysses, Harmondsworth, Penguin Books Ltd., 1968, p. 194. 
d'une absence; ce qui serait sa plénitude, au sujet, c'est, ce n'est que l'assentiment, la persistance qu'il revendique apposée en paraphe à l'ébranlement d'où il vint. Wo es war, soll Ich verden, encore. Le sujet projette dans son futur phallique le passé de perte pure qui le constituait, c'est ainsi qu'il gagne un présent, dans la rature ainsi effectuée de la perdurée devenant projet. C'est ainsi qu'il se déprend enfin de la perte éternelle, en la remettant en jeu sous les espèces du père, toujours à venir. Encore une bonne leçon du labyrinthe: d'Ariane au Minotaure, Thésée ne tient qu'à un fil: son désir, son futur, double hache parricide qu'il saura bien manier pour s'en sortir... du moins quelque temps.

Et c'est de s'être trop méfiés de ce fil qu'ainsi erreront à jamais, cloués incertains aux parois d'un labyrinthe désorienté, ces non-dupes auxquels on ne la fait pas, ces arpenteurs sans désir, ces encartés qui n'auront jamais eu de territoire. Ainsi erreront-ils...si du moins ils pouvaient tout à fait exister. Car de «non-dupe $"$ on ne connaît guère d'exemple, sinon peut-être, et parce qu'il est Dieu et parce qu'il est un bien étrange fils, le Christ qui ainsi, dans la logique lacanienne, serait le seul dont on puisse dire qu'il est une femme, puisque la femme ne peut exister que comme la toute qui serait Dieu... si Dieu existait.

Bref, le diable est aux vaches. Le diable, perversité foncière du signifiant qui nous tient d'être toujours ailleurs.

Mais, en fait d'animaux et de spectres, revenons à Ulysses, à un Shakespeare féru, à force d'études, d'Hamlet où il peut enfin tenir le rôle, jouer la partie, prendre le parti du fantômatique, du liquidé, du pire que père dans la mesure où il est condamné à revenir nous damner, Shakespeare, en vieux «buck ", affrontant d'un baptême assassin son rival «bucky * Burbage "who stands before him beyond the rack of cerecloth". "Qui lui fait face de l'autre côté du transparent" traduit assez pauvrement Morel. Or il faut savoir que ce "cerecloth" désigne en fait une toile d'embaumement et que "rack " est aussi, entre autres échafaudages, râteliers et porte-objets divers, un chevalet de torture. C'est donc du pilori qui le fait monstre, au sens étymologique, du sacrifice que, parlant d'au-delà du tombeau, au nom de la mort et à travers son voile, il fait de lui-même que Shakespeare, histrion suicidaire, dit le mot ( $"$ the words", il ne saurait y en avoir d'autres, l'article ici n'est surtout pas un déictique 
introductif à ce qui suit. Il dit au contraire le monumental du nom de mort qu'ils forment), la vérité, le «non-dupes errent : "Hamlet, I am thy father's spirit». Hamlet te dis-je du fond de ma mort projetée et de ce qu'il en reste: ce « spirit», eau de vie finalement, produit de la distillation de cette parole, de cette vérité. Tu ne peux être Hamlet que du leurre spectral qui de ma mort surgit devant toi. Accouchement par le père, miroir du suaire.

Aussi bien le pauvre Burbage est-il le sacrifié de ce piège démoniaque: le baptême oraculaire, le terrible *esprit du lieu * qui en scène sur lui se referme, c'est le signifiant d'où il se retrouve, pauvre dérisoire buck, effacé illico. Car, Shakespeare, en roi qui n'est pas roi, "to a son he speaks", c'est-à-dire un anonyme cloué du "non-dupes errent*, un anonyme qui peut être aussi bien "the son of his soul, the prince, young Hamlet" que "the son of his body, Hamnet Shakespeare ", mort à Stratford afin que vive celui qui porterait son nom. Car de toute façon c'est toujours au nom de la mort que parle le père, qu'il la relaie au nom du fils Hamnet ou qu'il la donne au nom de l'esprit à cet innocent de Burbage hamletisé. La mort en ce miroir. Le père, la mort, vérité de la structure constituante, défie le fils, ici sous les espèces de Burbage, d'oser parier l'assentiment (dire ici « l'assentiment à soi » serait une tautologie), le mouvement, la vie, pour échapper à cette chape embaumante et qui pue.

Joyce qui dans Ulysses organise notamment la rencontre eschatologique du fils Stephen Dedalus et du père Léopold Bloom, avec cette remarquable perversion de leurs patronymes qui fait porter au jeune Stéphane le «non-dupeserrent * par excellence, celui de l'inventeur de la machine à tuer les fils ${ }^{14}$ quand le juifissime errantissime Léopold se pare, lui, du nom propre de la germination: cette fleur, notamment de l'âge: "bloom of youth", Joyce donc a pris à son tour Shakespeare dans ses filets, il lui a fait ainsi le coup

14 C'est en effet une des leçons que l'on peut tirer du mythe de Dédale, exilé d'Athènes pour avoir précipité le fils de son frère, parce que le jeune homme devenait un peu trop son rival en architecture, du haut de l'Acropole, Dédale qui à Cnossos construit le labyrinthe où rituellement la fleur de la jeunesse d'Athènes, garçons et filles, autant dire la génération, est sacrifiée au Minotaure, Dédale enfin puni par son fils à son tour précipité quand le vieux malin croyait avec lui oublier son passé en effaçant son labyrinthe d'un trait d'ailes. Mais seul l'esprit du père peut voler. Les fils sont toujours condamnés à la colle, à la prothèse, ô phallus insaisissable! 
de Burbage, le coup du père, pour que vienne l'oeuvre, fils absolu, esprit de fils, perte mais jubilatoire, rivalité combinatoire, agonie salvatrice. Car c'est aussi le coup du "Chateaubriand ou rien ", du père au pire, pari d'où se fit Victor Hugo, que Joyce, avec Homère et Shakespeare (excusez du peu!), réédite avec le succès que l'on sait.

Le sujet, l'oeuvre aussi bien, est la suture d'une intersection de perte et de père. C'est pourquoi son lieu sera toujours délimité par un excès de matière sur le sens. C'est pourquoi accepter sa vérité sera toujours parier mais en passant par le coup du père, parier que le sens, dans cette asocialité fondamentale, ne peut advenir qu'ironisé à peine posé, "vulnérable au sortilège " seul, comme disait Baudrillard, féminisé peut-être, si la femme est bien a l'éternelle ironie" que dit Hegel et que Lacan répercute avec sa "pas toute », mais en tout cas charmé et déjoué par l'écriture, seule vérité du texte, seul oracle à surgir de ce lieu du non-lieu.

Professeur, comme de la vérité, ce que je sais de la littérature tient à ce que je suis prêt à accepter d'elle, à l'instant où sidérante elle surgit oracle dans ma voix, à l'instant, donc, où je ne sais plus ce que je dis, notamment parce qu'il n'est plus sûr alors que ce soit « je n qui parle. Mais, c'est une autre des leçons de Lacan, jamais ne suis-je plus maitre que dans ces moments-là, dans ces moments difficiles à faire venir, pour lesquels il me faut convoquer tout mon "métier", toute ma ruse, toute ma modestie, ces moments où la vérité de la littérature finit par surgir des cendres encore fumantes de mon petit ego professionnel. Le reste, tout le reste, n'est que savoir technique, irrémédiablement anodin. Comme un embarras de préparatifs, ce n'est qu'un balisage de l'espace où elle doit advenir, cette vérité de la littérature mais aussi cette vérité de tout le reste car s'«il y a d'l'Un " c'est bien de la vérité au sens du bon docteur Jacques-Marie. Elle ne m'adviendra que si ma parole professorale sait en être, au sens grec, « enthousiasmée».

Car la vérité, comme la cible au bout de l'arc zen, ne se vise que les yeux fermés. Et sa reconnaissance est avant tout acceptation de l'aléatoire de ce qui fait retour, retour impromptu d'une matière qui se rappelle ainsi au bon souvenir du sujet, mais pour qu'il en dispose.

Ainsi le «style» de Lacan, mallarméen, calembourgeois mais tel Bossuet quand un San Antonio le plume, non 
30

seulement manifeste-t-il l'indécidable de l'inconscient qui se fait voir (et se manifeste comme l'indécidable même) mais il provoque la pensée par une béance ouverte dans le langage même, béance organisée comme une chausse-trappe spéculaire qui oblige à considérer le rapport entre une inscription et ce que, de l'effacer de sa surcharge, elle cache, rapport pris dans une relation de simultanéité absolue, donc d'indécidable, rapport que l'on peut peut-être, mais c'est une autre histoire, assimiler à la « différance» derridienne.

Ce qui du moins m'apparait assez sûr c'est que, loin des technicités futiles de l'hyperbole littérale comme des grossières évanescences de l'expressivité, la théorie de la littérature n'aura sans doute jamais tant progressé que depuis qu'hors d'elle-même, Derrida avec la différance et Lacan avec son signifiant nomadisé, en un espace sans lieu ni borne, un dynamisme sans départ ni fin, l'auront pensée.

Qu'avons-nous donc encore à faire, littéraires sans honte ni alibi, du benoît impérialisme narratologique? L'essentiel n'est pas racontable, la spécificité de la littérature, sa vérité, n'est pas de récit. Mais de perte et de pari. Le texte est toujours à faire. Il (n')est (que) scriptible.

Et l'écriture demeure la seule vérité du texte, une vérité que nulle science jamais ne saurait atteindre. Car cette vérité ne s'avance, aléatoire et sauvage, que dans la nudité de sa matière toujours excédentaire. 